\title{
Evaluation of QT Interval Response to Marked RR Interval Changes Selected Automatically in Ambulatory Recordings
}

\author{
E Pueyo $^{1}$, P Smetana $^{2}$, M Malik $^{2}$, P Laguna $^{1}$ \\ ${ }^{1}$ Communications Technology Group, Aragón Institute of Engineering Research (I3A), \\ University of Zaragoza, Spain \\ ${ }^{2}$ Department of Cardiological Sciences, St. George's Hospital Medical School, London, U K
}

\begin{abstract}
A new approach was developed to evaluate the $Q T$ interval response to $R R$ interval changes in 24-hour ambulatory recordings, regarding different settings of the recording. First, a method was proposed to automatically identify beat positions in the following two situations: a) preceded by stable heart rate, $b$ ) after a sharp change in rate. Also, the evaluation across the complete recording was considered. For each recording, $Q T$ adaptation to $R R$ changes was independently assessed in the three described settings by using a global optimization technique that led to a characterization of the influence of a history of past $R R$ intervals on each QT measurement. Results showed that in stable heart rate situations, $Q T$ is mainly influenced by its immediately preceding $R R$ interval; however, in periods containing abrupt rate changes, a marked hysteresis effect was observed; this hysteresis was also noticeable in the 24-hour evaluation, although less manifest. Moreover, the time course of $Q T$ adaptation in response to sharp $R R$ changes derived in this study showed that there is a very rapid reaction during the first 50 seconds followed by a slower adjustment that takes nearly 2 minutes to complete.
\end{abstract}

\section{Introduction}

The relationship between the $Q T$ interval, measuring the time between the beginning of depolarization and the end of repolarization, and the heart rate $(H R)$ has been largely studied $[1,2]$. Several studies in the literature have approached the $Q T / R R$ relationship by selecting segments where cardiac rhythm is stable, and have then related each $Q T$ interval to its immediately preceding $R R$ interval $[3,4]$. However, when the $Q T / R R$ relationship is assessed in non-steady situations, where cardiac rhythm exhibits evident variations, it is necessary to take into account the hysteresis lag present in the $Q T$ adaptation to $R R$ changes [5]. The work by Lande et al. [6] illustrates this fact by showing that the $Q T / R R$ relationship is different when studied only over selected hysteresis-free segments and when no beat selection is made. The hysteresis phenomenon present in the $Q T$ interval response to $R R$ interval changes has been mainly investigated in controlled situations. In studies regarding sudden sustained changes in pacing rate [7, 8], two different phases in the adaptation process were observed: a fast one revealing the strong dependence of $Q T$ on the most recent $R R$ intervals, and a slow one evidencing a memory mechanism lasting for some minutes. Also, in recordings from exercise test protocols, Khran et al [9] showed that for long $Q T$ syndrome patients there is a continuous shortening of the $Q T$ interval during recovery from exercise, which is clearly interpreted as a hysteresis effect in the adaptation to rate changes. In situations with spontaneous heart rate variations, like those found in ambulatory 24-hour recordings, the assessment of the $Q T$ interval dependence on past $R R$ interval changes is more complicated, due to the continuous shortening and lengthening of the intervals. Besides, evaluation over long periods, such as 24 hours, has the drawback of analyzing at the same time segments where cardiac rhythm is basically stable and others where substantial heart rate changes are present. In our work, we propose a method for selecting segments of the recording that contain sharp $R R$ transitions and, over such selected segments, individual adaptation profiles characterizing the $Q T$ dependence on previous $R R$ intervals are derived. Such hysteresis profiles are compared with the ones obtained from the evaluation on stable heart rate segments, also selected automatically in the present study, and on the complete 24-hour recording. With the proposed methodology, it is also possible to determine, for each recording, the different phases of the adaptation process.

\section{Methods}

\subsection{Population and data preparation}

The study evaluated 24-hour 3-lead Holter ECG recordings obtained from 939 patients of the EMIAT trial [10] that investigated survivors of acute myocardial 
infarction and randomized them to treatment with amiodarone or placebo. In each recording, $Q T$ and $R R$ intervals were measured automatically in each lead using commercial equipment (Pathfinder700, Reynolds Medical, Hertford, U.K.). For each lead of each recording, only cardiac cycles for which the electrocardiograph was able to determine $Q T$ and $R R$ interval measurements were considered. Besides, visual inspection was applied on the $Q T$ series in order to detect possible errors in the automatic delineation and then manual correction was applied in such cases. Subsequently, the lead with most accepted measurements was selected for each recording. Anomalies caused by QRS detector errors and by ectopic beats were identified using a previously proposed strategy [11] and the corresponding positions were rejected from the analysis.

\subsection{Selection of stable and unstable heart rate segments}

From the $Q T_{i}$ and $R R_{i}$ series containing all the available valid measurements of $Q T$ and $R R$ intervals, new signals $Q T(n)$ and $R R(n)$ were obtained by linear interpolation at sampling frequency of $1 \mathrm{~Hz}$. The $Q T(n)$ and $R R(n)$ signals were low-pass filtered with a Butterworth secondorder filter (cut-off frequency $0.03 \mathrm{~Hz}$ ), generating $Q T_{f}(n)$ and $R R_{f}(n)$ series.

On the $R R_{f}(n)$ signal, the variance was measured in 300 -second segments sliding every 15 seconds, obtaining the series $\sigma_{R R}^{2}(m)$, with $m$ denoting the consecutive number of a 300-second segment. Subsequently, two different thresholds $u$ and $v$, later described, were applied to the $\sigma_{R R}^{2}(m)$ series along the 24-hour recording (Fig. 1).
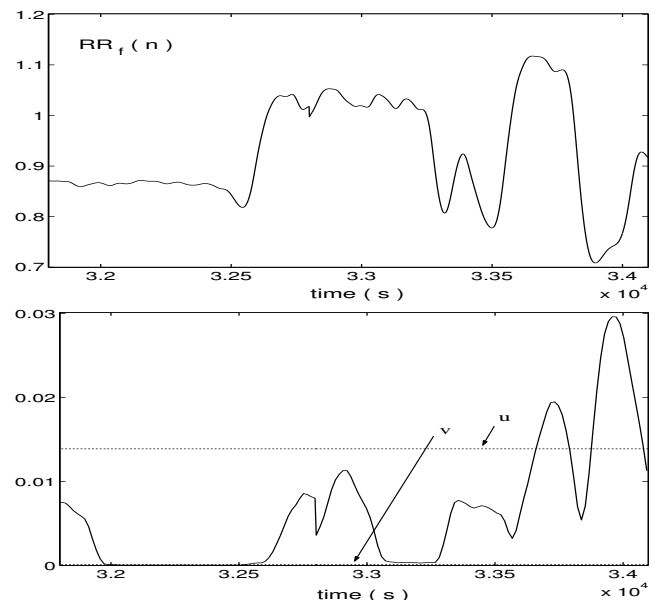

Figure 1. $\quad R R_{f}(n)$ signal (top pannel) and its variance $\sigma_{R R}^{2}(m)$ with thresholds $u$ and $v$ defined from $2 \%$ and $98 \% \sigma_{R R}^{2}(m)$ percentiles, respectively (bottom panel).

Episodes of sudden sustained heart rate changes were determined by identifying positions of cardiac cycles corresponding to preceding 300-second segments in which $\sigma_{R R}^{2}(m) \geq u$. Analogously, stable heart rate periods were selected according to the condition $\sigma_{R R}^{2}(m) \leq v$.

The thresholds $u$ and $v$ were selected individually for each recording, since the range of $R R$ variations differed substantially among subjects. To evaluate the same number of states in the two analyzed cases (stable and unstable heart rate), $u$ and $v$ were defined from the percentiles of $\sigma_{R R}^{2}(m)$ at $2 \%$ and $98 \%$, respectively.

\subsection{Individualized QT/RR hysteresis profiles}

In the subsequent analysis, only beat positions with valid $Q T$ measurements and preceded by a 300 -second window containing all valid $R R$ measurements were considered. For each recording, $Q T / R R$ hysteresis profiles were determined separately in three different scenarios: on the one hand, the selected set of beats preceded by stable heart rate; on another hand, selected beats preceded by marked heart rate changes; and, finally, considering the complete 24-hour recording. In each case, $Q T$ interval dependence on preceding $R R$ intervals was characterized by optimum weighted averages of $R R$ interval measurements in a window of 300 seconds $(\overline{R R})$, with optimum defined as leading to minimum regression residual of the $[Q T, \overline{R R}]$ fit. In order to derive such an optimum weight distribution independently for each setting and recording, a global optimization algorithm based on the Direct method [12, 13] was implemented, in which the objective function to be minimized was defined at each weight vector $\mathbf{w}=$ $\left(w_{-N+1}, \ldots, w_{0}\right)$ as the global residual from fitting any of 10 a-priori selected regression models [14] to the $\left[Q T_{i}, \overline{R R}_{i}\right]$ data, with $\overline{R R}_{i}$ computed for each $i$-th beat as

$$
\overline{R R}_{i}=\sum_{j=-N+1}^{0} w_{j} R R_{i+j},
$$

In the above expression, $N$ is the mean number of beats contained in 300-second windows preceding the analyzed positions and $w=\left(w_{-N+1}, \ldots, w_{0}\right)$ are all positive and normalized such that $w_{-N+1}+\ldots+w_{0}=1$.

As a result, 10 different combinations of weights $w_{j}$ and regression parameters were determined for each recording and setting, each combination characterizing the optimum $R R$ influence associated with one regression model. A unique pattern of averaging window was identified by selecting the model leading to the minimum residual.

\subsection{Adaptation phases}

The weight curves obtained in section 2.3 characterizing the $Q T / R R$ hysteresis phenomenon, particularly in response to sharp heart rate changes, allowed the study of 
different phases in the $Q T$ adaptation. From the hysteresis profiles individually determined, it could be observed that in most cases the adaptation comprised two clearly definite phases. In order to quantify the contribution of each separate phase to the whole adaptation process, we first calculated, for each recording, the minimum window length $L_{90}$ required to achieve $90 \%$ of the sum of weights, so as to avoid considering cardiac cycles that do not effectively affect the $Q T$ interval. From the individual weight curve extended over such minimum length, we initially searched for points in which the first derivative changed polarity to identify possible transitions between periods with different adaptation rates. When no separated periods were identified by the above criterion, we determined a change in the adaptation rate by searching for a maximum in the second derivative of the weight curve.

Each of the previously identified phases in the $Q T$ adaptation, which we denoted by $P_{k}$, was independently fitted with an exponential model (Fig. 2):

$$
w(j)=\exp \left(A_{k} j+B_{k}\right), j \in P_{k} .
$$

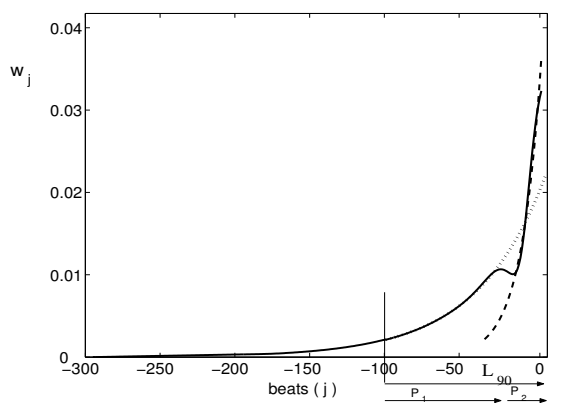

Figure 2. For derivation of the different $Q T$ adaptation phases, points marking significant changes in the adaptation rate were identified (see text for details). In order to characterize each phase separately, exponential curves were fitted to each of them.

\section{Results}

The influence of heart rate on the $Q T$ interval, evaluated separately over selected beats preceded by stable and unstable heart rate, led, as expected, to very different adaptation profiles for the two situations. While in segments where the $R R$ signal was basically constant, $Q T$ was found to be mainly influenced by the immediately preceding cardiac cycle, the assessment over segments with marked heart rate transitions showed a pronounced hysteresis effect in the $Q T / R R$ adaptation. The evaluation over the 24 hours also showed an evident $Q T$ lag in response to $R R$ changes, although it was less manifest than in situations of abrupt rate changes (Fig. 3). In both settings, such a dependence relation was found to be highly individual, but in any case results confirmed the necessity of taking into account the influence of a considerable number of cardiac cycles when investigating the $Q T / R R$ relationship. Quantifying the effective influence of past

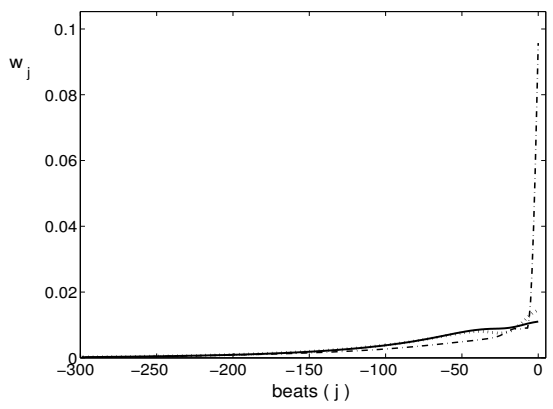

Figure 3. Mean optimum weight distributions averaged over patients. The dotted line shows the results derived from 24-hour evaluation, while the solid line corresponds to abrupt rate changes analysis and the dashed line is for stable heart rate segments.

$R R$ intervals on each $Q T$ measurement in terms of the time for $90 \%$ adaptation $\left(L_{90}\right)$ from the mean weight distributions, we found that just 8 seconds were required when assessment was done over stable heart rate situations, while 148 seconds were found in the response to marked alterations in heart rate and 109 seconds when evaluated over the complete 24-hour recordings.

The study of the different phases in the $Q T$ response to marked $R R$ changes revealed that for the vast majority of patients (932 out of 939), two main distinct stages composed the adaptation process, while only 7 patients presented a clear monophasic adaptation. Regarding the 99\% of patients with two adaptation phases, it could be corroborated that $Q T$ presents an initial fast reaction to $R R$ changes that lasts, in mean, for around 50 seconds, and a posterior slower response that is prolonged for nearly 2 minutes. These two different adaptation steps were also quantified in terms of the parameters $A_{k}$ (velocity) and $B_{k}$ (ordinate) from the exponential fits. Despite the wide range of values covered both by $A_{k}$ and $B_{k}$ due to the highly individual $Q T / R R$ adaptation profiles previously described, we could characterize a very steep initial adaptation with mean velocity $A_{2}$ of $35.7 \mathrm{~ms}^{-1}$ and mean ordinate $B_{2}$ of -8.4 , followed by a delayed adjustment expressed, in mean, by $A_{1}=17.5 \mathrm{~ms}^{-1}$ and $B_{1}=-6.4$. Correlation values above 0.9 confirmed the suitability of the exponential models for this purpose.

\section{Discussion and conclusions}

Results from our study confirmed that when $Q T / R R$ relationship is investigated and potentially used to correct the $Q T$ interval for the effects of heart rate, it is fundamental to take into account the influence of a history of past $R R$ intervals on each $Q T$ measurement, unless the 
analysis is limited to selected ECG segments or recordings where cardiac rhythm presents high stability. Such a hysteresis phenomenon present in the $Q T$ adaptation to spontaneous $R R$ changes is more manifest when assessed only over selected beats preceded by sharp heart rate changes than when the evaluation is carried out across the complete recording, the reason being that along the 24 hours there are both segments where cardiac rhythm is almost constant and others where it is very unsteady, and only the last ones have a significant contribution to the observed hysteresis.

Moreover, in our analysis it has been shown that the $Q T$ interval responds to a major change in rate in two different phases, which is consistent with the results reported by Lau et al [8] on the response of ventricular paced $Q T$ interval to abrupt changes in pacing rate and with the findings reported by Franz et al [7] on the adaptation of endocardial monophasic action potential to changes in pacing rate. In our work, it was found that the $Q T$ adaptation to sharp spontaneous $R R$ interval changes can be described by an initial fast phase, which covers around 50 seconds (in mean) and is approapriately modelled by an exponential curve with very steep gradient, and a second phase that is considerably slower and takes longer time to complete. The study of the time course of $Q T$ adaptation in response to abrupt rate changes might prove to be useful for the evaluation of the potential modifications induced by antiarrhythmic drugs such as amiodarone.

Finally, an important finding of our study is referred to the high inter-subject variability found not only in the $Q T / R R$ adaptation times but also in the hysteresis profiles, both when assessed in response to abrupt rate changes or from the 24-hour recordings. The individually determined weighted averaged $R R$ measures $(\overline{R R})$ proposed in our work aim to provide values able to relate each $Q T$ interval with heart rate taking into account the lag present in the $Q T$ interval adaptation to $R R$ interval changes.

\section{Acknowledgements}

This work was supported by projects TIC2001-2167-C02-02 from MCYT/FEDER and P075/2001 from CONSID-DGA (Spain).

\section{References}

[1] Bazett JC. An analysis of time relations of electrocardiograms. Heart 1920;7:353-370.

[2] Fridericia LS. Die systolendauer im elekrokardiogramm bei normalen menschen und bei herzkranken. Acta Med Scand 1920;53:469-486.

[3] Kligfield P, Lax KG, Okin PM. QT interval-heart rate relation during exercise in normal men and women: definition by linear regression analysis. J Am Coll Cardiol 1996;28:1547-1555.
[4] Karjalainen J, Viitasalo M, Manttari M, Manninen V. Relation between QT intervals and heart rates from 40 to 120 beats/min in rest electrocardiograms of men and a simple method to adjust QT interval values. J Am Coll Cardiol 1994;23:1547-1553.

[5] Alessandrini RS, McPherson DD, Kadish AH, Kane BJ, Goldberger JJ. Cardiac memory: a mechanical and electrical phenomenon. Am J Physiol 1997;272:952-959.

[6] Lande G, Funck-Brentano C, Ghadanfar M, Escande D. Steady-state versus non-steady-state QT-RR relationships in 24-hour Holter recordings. Pacing Clin Electrophysiol 2000; 23:293-302.

[7] Franz M. R.and Swerdlow CD, Liem LB, Schaeffer J. Cycle length dependence of human ventricular action potential duration in steady and non-steady state. In Butrous GS, Schwartz PJ (eds.), Clinical aspects of ventricular repolarization. London: Farrand Press, 1989; 163-174.

[8] Lau CP, Freedman AR, Flemming S, Malik M, Camm AJ, Ward DE. Hysteresis of the ventricular paced QT interval in response to abrupt changes in pacing rate. Cardiovasc Res 1988;22:67-72.

[9] Krahn AD, Yee R, Chauhan V, Skanes AC, Wang J, Hegele RA, Klein GJ. Beta blockers normalize QT hysteresis in long QT syndrome. Am Heart J 2002;143:528-534.

[10] Julian DG, J CA, Frangin G, Janse MJ, Munoz A, Schwartz PJ, Simon P. Randomised trial of effect of amiodarone on mortality in patients with left-ventricular dysfunction after recent myocardial infarction: EMIAT. European Myocardial Infarct Amiodarone Trial Investigators. Lancet 1997; 349:667-674.

[11] Mateo J, Laguna P. Analysis of heart rate variability in the presence of ectopic beats using the heart timing signal. IEEE Trans Biomed Eng 2003;50:334-343.

[12] Jones DR, Perttunen CD, Stuckman BE. Lipschitzian optimization without the Lipschitz constant. Journal of Optimization Theory and Applications 1993;79:157-181.

[13] Lewis RM, Torczon V, Trosset MW. Direct search methods: then and now. Journal of Computational and Applied Mathematics 2000;124:191-207.

[14] Pueyo E, Smetana P, Hnatkova P, Laguna P, Malik M. Time for QT adaptation to RR changes and relation to arrhythmic mortality reduction in amiodarone-treated patients. In Computers in Cardiology 2002. Memphis: IEEE Computer Society Press, 2002; 565-568.

Address for correspondence:

Esther Pueyo

Communications Technology Group / Aragón Institute of Engineering Research (I3A) / University of Zaragoza

María de Luna 3 / 50018 Zaragoza / Spain

tel./fax: ++34-976-762704/2111

epueyo@unizar.es 